\title{
SHORT REPORT \\ Cryptosporidium hominis genotypes involved in increased incidence and clusters of cases, Navarra, Spain, 2012
}

\author{
I. FUENTES ${ }^{1}$, C. MARTÍN ${ }^{2}$, X. BERISTAIN ${ }^{2}$, A. MAZÓN ${ }^{2}$, J. M. SAUGAR ${ }^{1}$, \\ A. BLANCO ${ }^{1}, \mathrm{M}_{\text {. GARCÍA CENOZ }}^{3,4}$, M. VALLE-CRISTIA ${ }^{3,4}$, C. EZPELETA $^{2}$ \\ AND J. CASTILLA ${ }^{3,4 *}$ \\ ${ }^{1}$ Servicio de Parasitología, Centro Nacional de Microbiología, Instituto de Salud Carlos III, Majadahonda, Spain \\ ${ }^{2}$ Servicio de Microbiología Clínica, Complejo Hospitalario de Navarra, Pamplona, Spain \\ ${ }^{3}$ Instituto de Salud Pública de Navarra, Pamplona, Spain \\ ${ }^{4}$ CIBER Epidemiología y Salud Pública, Spain
}

Received 19 July 2013; Final revision 23 June 2014; Accepted 25 June 2014;

first published online 14 July 2014

\section{SUMMARY}

Two clusters of confirmed cryptosporidiosis infections were detected in Navarra, Spain, in the summer of 2012, in the context of an increased incidence in the region. Molecular subtyping of Cryptosporidium hominis determined that one cluster, occurring in an urban area, was due to the predominant circulating subtype IbA10G2R2 and the other cluster, with cases occurring in a rural area, was due to a rare subtype IaA18R3. No single exposure was associated with infection, although exposure to certain children's pools was reported by a majority of patients interviewed in each cluster. Genotyping tools were useful in the investigation and could aid investigation of cryptosporidiosis outbreaks in Spain in the future.

Key words: Cryptosporidium, epidemiology, outbreaks, surveillance, waterborne infections.

Cryptosporidium spp. outbreaks have been associated with contaminated drinking water [1], swimming pools [2], contact with farm animals [3], day-care centres and other institutions [4] or consumption of contaminated food and drink [5].

In late summer 2012 a high incidence of cryptosporidiosis was reported in The Netherlands, UK and Germany, but no single source could explain it [6]. Cryptosporidiosis was not a reportable disease in Spain in 2012. In Navarra, a region in northern Spain with 644566 inhabitants, the samples for parasitological diagnosis of patients attending hospitals and primary-care centres were processed in the same

\footnotetext{
* Author for correspondence: Dr J. Castilla, Instituto de Salud Pública, Leyre 15, 31003 Pamplona, Spain.

(Email: jcastilc@navarra.es)
}

laboratory. A qualitative immunochromatographic test (CerTest Crypto-Giardia ${ }^{\circledR}$, CerTest Biotec, Spain) was used to detect antigens of Cryptosporidium spp. and Giardia intestinalis in stool samples of patients aged $<15$ years for whom a parasite analysis was requested. In this region an increased incidence of cryptosporidiosis was detected during the second half of 2012.

Between July and December 2012, a total of 162 patients with diarrhoea due to Cryptosporidium spp. were confirmed, compared to nine cases during the first half of the year. The percentage of patients confirmed for Cryptosporidium spp. among all those tested in the second half of the year increased from $5 \cdot 0 \%(49 / 977)$ in 2011 to $11 \cdot 3 \%$ in $2012(162 / 1435$, $P<0 \cdot 001)$. Of the 162 patients in the second half of 2012, 66\% were males, and $98(60 \%)$ were clustered in a 4-week period (20 August-16 September). 


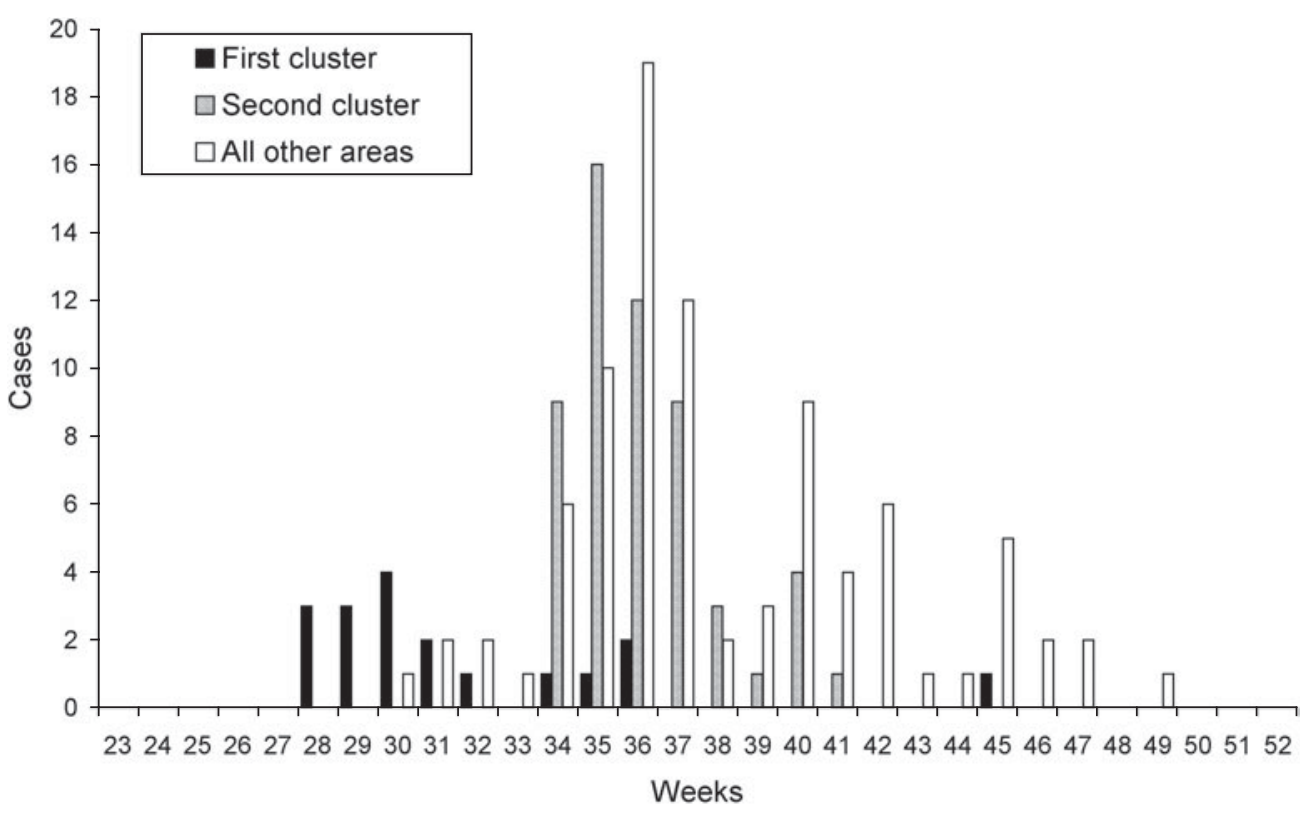

Fig. 1. Weekly confirmed cases of cryptosporidiosis in the two municipalities with clusters and in the rest of the region, Navarra, Spain, June-December 2012.

All confirmed cases occurred in patients aged 0 14 years, and 124 (77\%) were aged 1-4 years. Only one patient required hospitalization. Although cases were confirmed in 28 of the 56 health districts of Navarra, two clusters - defined as a statistically significant higher incidence than expected of laboratory-confirmed cases in a geographical area or municipality - were identified (Fig. 1, Table 1).

Fifty positive samples were sent to the National Microbiology Centre for confirmation by direct immunofluorescence (DIF) test for detection of Cryptosporidium oocysts and Giardia cysts (Crypto/ Giardia Cel ${ }^{\circledR}$, Cellabs, Australia) and molecular characterization. DNA was extracted directly from faecal samples using QIAamp DNA stool Mini kit (Qiagen, Germany). In 40 samples the predominant Cryptosporidium species could be determined by nested polymerase chain reaction-restriction fragment length polymorphism of the Cryptosporidium oocyst wall protein (COWP) gene [7] and subtyping using gp60 gene amplification sequencing [8].

The first cluster consisted of 18 Cryptosporidiumpositive cases in the 1019 children aged $<15$ years $(1.77 \%)$ in a rural town, with 12 of these cases in the 256 children aged $1-4$ years $(4 \cdot 69 \%)$. Thirteen patients presented between 13 July and 7 August. Epidemiological interviews were conducted with the parents of 10 children. In the 12 days before symptom onset, six children had bathed in the only public swimming pool for young children in the town, seven had attended the town's day-care centre, and one had not been to any of these places but had been in contact with one other confirmed case. The seven Cryptosporidium isolates characterized were identified as C. hominis subtype IaA18R3 (Table 1).

The second cluster occurred between 20 August and 5 October, and comprised 55 Cryptosporidiumpositive children living in an urban municipality (11 524 inhabitants), with 46 patients clustered in the first 4 weeks. The patients' ages ranged from 0 to 8 years, and 52 patients $(95 \%)$ were aged $1-4$ years. The incidence rate in the municipality was $2.13 \%$ in children aged $<15$ years, and $4 \cdot 45 \%$ in those aged 1-4 years. The parents of 33 patients were interviewed. In the 2 weeks before symptom onset, $30(91 \%)$ patients had bathed in the municipal children's pool, eight $(24 \%)$ had bathed in other children's pools, ten $(30 \%)$ had attended one of the municipal day-care centres, and nine $(27 \%)$ had been on vacation outside the region, one of them for the entire incubation period. In eight cases $(24 \%)$, other household members had the same symptoms. The 28 Cryptosporidium isolates characterized were identified as $C$. hominis subtype IbA10G2R2 (Table 1).

In both clusters the peak incidence was preceded by very hot weeks during which swimming pool use was high as noted in local newspapers. After the detection of each cluster all the water in the pools related to cases was immediately emptied and the pools were cleaned. Inspection of the pools revealed no 
Table 1. Characteristics of Cryptosporidium-positive patients clustered in the two municipalities and in the rest of the region, Navarra, Spain, July-December 2012

\begin{tabular}{llll}
\hline \hline & First cluster & Second cluster & All other cases \\
\hline Place & Rural town & Urban municipality & Rural and urban areas \\
Number of cases & 18 & 55 & 89 \\
Age range, years & $0-14$ & $0-8$ & $1-12$ \\
Percentage of patients aged 1-4 years & $67 \%$ & $95 \%$ & $67 \%$ \\
Male & $16(89 \%)$ & $36(65 \%)$ & $55(62 \%)$ \\
Attack rate in children aged 0-14 years & $1 \cdot 77 \%$ & $2 \cdot 13 \%$ & $0 \cdot 09 \%$ \\
Attack rate in children aged 1-4 years & $4 \cdot 69 \%$ & $4 \cdot 45 \%$ & $0 \cdot 23 \%$ \\
Detection of first and last cases & $13 \mathrm{July}-11$ Nov. & 20 Aug.-5 Oct. & 23 July-9 Dec. \\
Week with the highest incidence & $23-29$ July & 27 Aug.-2 Sept. & $3-9$ Sept. \\
Bathing in the municipal children's pool & $6 / 10(60 \%)$ & $30 / 33(91 \%)$ & n.d. \\
Attendance at day-care centres & $7 / 10(70 \%)$ & $10 / 33(30 \%)$ & n.d. \\
Family members with symptoms & $2 / 10(20 \%)$ & $8 / 33(24 \%)$ & n.d. \\
Number of Cryptosporidium isolates characterized & 7 & 28 & 5 \\
Cryptosporidium species identified & $C$. hominis & $C$. hominis & C. hominis \\
Cryptosporidium family and subtypes identified & IaA18R3 & IbA10G2R2 & IbA10G2R2 and A14R3 \\
\hline \hline
\end{tabular}

n.d., Epidemiological interviews not done.

anomalies, and the chlorine levels were correct in all the daily tests, although these tests did not include Cryptosporidium detection. The records of the daily monitoring of the public water supply were reviewed and no signs of contamination or lapses in chlorination by chemical testing were found. No food or drink common to the majority of cases was found.

During the second half of 2012, while these clusters were occurring, another 89 Cryptosporidium-positive patients were detected in other municipalities of Navarra. The rate of confirmed patients in these other municipalities was $0.09 \%$ in children aged $<15$ years, rising to $0 \cdot 23 \%$ in children aged $1-4$ years. Cryptosporidium isolates could be characterized in five of these patients; four corresponded to $C$. hominis IbA10G2R2 and one to C. hominis A14R3 (Table 1).

Parents of children with cases of cryptosporidiosis were urged to ensure that all household members washed their hands frequently, and it was recommended that children should not attend the day-care centre or bathe in swimming pools until 1 week after symptoms had resolved.

In summary, in the second half of 2012 Cryptosporidium spp. was confirmed in $4-5 \%$ of the children aged $1-4$ years in two municipalities and $0 \cdot 2 \%$ in the rest of Navarra. During the same period, increased incidence was also reported in other European countries [6]. Most of the Cryptosporidium isolates characterized in Navarra corresponded to C. hominis IbA10G2R2, which was also the predominant circulating subtype in Europe [6] and Australia [9]. These findings suggest that what was observed in Navarra may be the local manifestation of a much wider circulation of this subtype of $C$. hominis.

C. hominis subtype IaA18R3, found in cases of the first cluster, is an infrequent subtype, which has not been found in other European countries but has been reported in children in Bangladesh [10].

Adult samples were not tested for Cryptosporidium antigen, nor were stool samples available for all symptomatic children; therefore the cases diagnosed are likely to represent only part of the total number of persons affected. Early in both clusters, a single transmission source was considered unlikely since we could not identify an exposure capable of explaining the origin of all the cases. No controls were interviewed, which would have made it possible to test hypotheses about potential factors involved in the transmission. We suggest that the most plausible explanation is that various sources were involved in the transmission. The risk factors most frequently reported by the parents were bathing in certain children's pools, attendance at certain day-care centres and living in a household with others who had similar symptoms. Furthermore, some patients may have contracted the infection outside the region. The swimming pool routine control checks were performed correctly, but we cannot rule out possible transitory contamination of the water in the children's pools. Other studies have shown that the usual quality control checks in water for recreational use may not be sufficient to prevent transmission of Cryptosporidium [2]. 
Few published studies have characterized human cryptosporidiosis in Spain, and this is the first report of clusters of Cryptosporidium cases describing the species and genotypes.

The increase in cryptosporidiosis in Europe highlights the need for wider surveillance. Genotyping and subtyping tools are important complementary components in the epidemiological investigation of the situation and could aid investigation of cryptosporidiosis outbreaks in Spain in the future.

\section{ACKNOWLEDGEMENTS}

We thank the primary healthcare and hospital personnel for assistance in specimen collection, and Begoña Bailo and María Aguilera for their technical assistance. This study was supported in part by a grant of the Instituto de Salud Carlos III (FIS PI10/01240).

\section{DECLARATION OF INTEREST}

None.

\section{REFERENCES}

1. MacKenzie WR, et al. A massive outbreak in Milwaukee of Cryptosporidium infection transmitted through the public water supply. New England Journal of Medicine 1994; 331: 161-167.

2. Insulander $\mathbf{M}$, et al. An outbreak of cryptosporidiosis associated with exposure to swimming pool water. Scandinavian Journal of Infectious Diseases 2005; 37: 354-360.

3. Smith RP, et al. Investigation of farms linked to human patients with cryptosporidiosis in England and Wales. Preventive Veterinary Medicine 2010; 94: 9-17.

4. Vandenberg O, et al. Management of a Cryptosporidium hominis outbreak in a day-care center. Pediatric Infectious Diseases Journal 2012; 31: 10-15.

5. Quiroz ES, et al. An outbreak of cryptosporidiosis linked to a food handler. Journal of Infectious Diseases 2000; 181: 695-700.

6. Fournet N, et al. Simultaneous increase of Cryptosporidium infections in the Netherlands, the United Kingdom and Germany in late summer season, 2012. Eurosurveillance 2013; 18(2): pii=20348.

7. Pedraza-Díaz S, et al. Nested polymerase chain reaction for amplification of the Cryptosporidium oocyst wall protein gene. Emerging Infectious Diseases 2001; 7: 49-56.

8. Feltus DC, et al. Evidence supporting zoonotic transmission of Cryptosporidium spp. in Wisconsin. Journal of Clinical Microbiology 2006; 44: 4303-4308.

9. Waldron LS, et al. Molecular epidemiology and spatial distribution of a waterborne cryptosporidiosis outbreak in Australia. Applied Environmental Microbiology 2011; 77: 7766-7771.

10. Hira KG, et al. Genetic diversity of Cryptosporidium spp. from Bangladeshi children. Journal of Clinical Microbiology 2011; 49: 2307-2310. 